\title{
Giant Emission Structures and Dust-poor Holes around Planetary Nebulae on IRAS SkyView Images?
}

\author{
Ronald Weinberger \\ Institut für Astrophysik, Leopold-Franzens Universität Innsbruck, \\ Technikerstraße 25, A-6020 Innsbruck, AUSTRIA
}

\begin{abstract}
A systematic search of the wider neighborhood of Planetary Nebulae (PNe) on IRAS SkyView images revealed structures like bipolar dust blobs, curved filaments and dust-poor cavities which in several cases suggest that they are genuine and not just due to projection effects.
\end{abstract}

As a result of a systematic search around about 400 Galactic PNe on IRAS SkyView maps I found a number of emission structures as well as holes. Only few IRAS structures of such a type are known: One example is the arc in the south-west of NGC 3242 which is present also at 60 and $100 \mu \mathrm{m}$ (Meaburn et al. 2000), another a $100 \mu \mathrm{m}$ cavity around NGC 4361 (Weinberger 1999). Any claim to have discovered extended structures on these maps and, particularly, such with tremendous dimensions as we did - like a $3.2 \mathrm{pc} 12 \mu \mathrm{m}$ nebula around NGC 1514 and dust arcs 14 pc away from NGC 6826 - must face a significant objection: Are such structures real or just chance projections? Statistics does not help much given the presence of all kinds of cavities, filaments, arcs, wisps etc. visible on SkyView maps. Hence, chance coicidences can occur and more than just one positional coincidence is required. In several cases we found a PN being close to the midpoint of lines joining one or more dust blobs, e.g. for Lo 4. Close means that the predecessor of the PN must have a marked velocity relative to the ambient medium. Interestingly, in these cases the $\mathrm{PNe}$ shells and/or halos show clear signs of interaction just where one expects them to be. Such coincidences can be regarded as rather strong arguments for the reality of the partly huge structures. It is beyond question that such huge structures, if real, are of importance: Since a blob, say 5 pc away from the PN will probably have been ejected in the pre-PN phase, one gets insights in the mass-losing process during the AGB phase, for which spherical mass-loss is usually assumed. Further the problem why structures can survive $10^{5}$ years must be addressed. Generally, these structures should be much more frequent, since many of them will be hidden from view because of contamination by infrared emission from dust in the background. If so, then the mass outflows during the AGB phase are of some relevance for the ISM not only with respect to enrichment, but also as to dynamical interactions.

\section{References}

Meaburn, J., Lopez, J.A., \& Noriega-Crespo, A. 2000, ApJS, 128, 321

Weinberger, R. 1999, AG Abstr. Ser., 15, 65 